See discussions, stats, and author profiles for this publication at: https://www.researchgate.net/publication/311756931

\title{
Compliant control for soft robots: Emergent behavior of a tendon driven anthropomorphic arm
}

Conference Paper · October 2016

DOI: 10.1109/ROS.2016.7759138

CITATIONS

7

4 authors, including:

Georg Martius

Max Planck Institute for Intelligent Systems

67 PUBLICATIONS 399 CITATIONS

SEE PROFILE

6re)

Ralf Der

134 PUBLICATIONS 1,739 CITATIONS

SEE PROFILE

Some of the authors of this publication are also working on these related projects:

Project The Human Brain Project: Neurorobotics View project
READS

113

75. Rafael Hostettler

15 PUBLICATIONS 73 CitATIONS

SEE PROFILE 


\title{
Compliant control for soft robots: emergent behavior of a tendon driven anthropomorphic arm*
}

\author{
Georg Martius ${ }^{1}$, Rafael Hostettler ${ }^{2}$, Alois Knoll ${ }^{2}$, and Ralf Der ${ }^{3}$
}

\begin{abstract}
With the accelerated development of robot technologies, optimal control becomes one of the central themes of research. In traditional approaches, the controller, by its internal functionality, finds appropriate actions on the basis of the history of sensor values, guided by the goals, intentions, objectives, learning schemes, and so forth. While very successful with classical robots, these methods run into severe difficulties when applied to soft robots, a new field of robotics with large interest for human-robot interaction. We claim that a novel controller paradigm opens new perspective for this field. This paper applies a recently developed neuro controller with differential extrinsic synaptic plasticity to a muscle-tendon driven arm-shoulder system from the Myorobotics toolkit. In the experiments, we observe a vast variety of self-organized behavior patterns: when left alone, the arm realizes pseudorandom sequences of different poses. By applying physical forces, the system can be entrained into definite motion patterns like wiping a table. Most interestingly, after attaching an object, the controller gets in a functional resonance with the object's internal dynamics, starting to shake spontaneously bottles halffilled with water or sensitively driving an attached pendulum into a circular mode. When attached to the crank of a wheel the neural system independently develops to rotate it. In this way, the robot discovers affordances of objects its body is interacting with.
\end{abstract}

\section{INTRODUCTION}

The demand of creating robots that are capable of becoming part of our everyday lives and our society is rising. However, the control of such highly complex autonomous robots is a challenging task. Robots mimicking the human morphology, also called anthropomimetic robots, are a prominent example of this challenge. To model an essential mechanical aspects of the human musculoskeletal system these robots are driven by artificial muscles and tendons. This makes them lighter and softer than classical systems and thus saver to interact with. These properties make them favorable for service robots in human environments. Moreover, because of their human-like morphology, they can be used for better understanding human behavior generation and development. Since muscles are the active part in the body, they are also the most complex part to model. To date, in humanoid anthropomimetic robots, these muscles are either pneumatic or consisting of a motor coiling a tendon, often in series

* GM received funding from the People Programme (Marie Curie Actions) of the European Union's Seventh Framework Programme (FP7/2007-2013) under REA grant agreement no. [291734].

${ }^{1}$ IST Austria, Am Campus 1, 3400 Klosterneuburg, Austria gmartiuseist.ac.at

2Institut für Informatik VI, TU München, Boltzmannstr. 3, 85748 Garching bei München, Germany $\{$ hostettl|knoll\}@in.tum. de

${ }^{3}$ Max Planck Institute for Mathematics in the Science, Inselstr. 22, 04103 Leipzig, Germany ralfder@mis.mpg.de with an elastic element. This simple construction exhibits many of the properties of biological muscles: they can only pull, the transmitted force is depending on the current joint angle through a changing lever arm, the tendons wrap around bones, can be multiarticular and the system is often under-constrained and as such, there are many solutions to every desired joint position. This approach also introduces a number of non-biological constraints, such as hysteresis through friction in the tendon routing, tendon elasticity and bandwidth limitations from the motors. Lastly, certain features of biological musculoskeletal systems are omitted. Most notably, these are ligaments, elongated muscles attachments and effects from muscle bulging.

World wide, several of such muscle tendon driven systems have already been built. While mechatronically at an advanced level, the control of such systems is still in its infancy. In principle, any of the known control paradigms can be used for the behavior generation. To date, the two branches of robotics - the classical AI versus the embodied approachcoexist, each one having its realm of relevance. Combining them using learning approaches becomes more and more successful. On the classical side, the DARPA challenge presents numerous examples of progress but reveal also a realm of failures of these systems even under remote control. The embodied approach seems to be favored in systems with strong physical effects, like soft robotic systems, where the engineering approaches run into severe difficulties. Somewhere in between are a variety of new control paradigms, best demonstrated by the amazing locomotion abilities of the Boston dynamics robots, like BigDog, Petman and others. These are ingeniously engineered systems for realizing a specific set of tasks with their highly specialized bodies.

The limitations of present day engineering approaches to human like structures is best seen when considering muscle-tendon driven (MTD) systems where an important line of research was shaped by EU projects leading from Cronos, to EcceRobot to Myorobotics, but also by Japanese projects creating the highly sophisticated Kenshiro robot [1]. While excellent work has been done in planning, constructing, and eventually building these robots [2], the control of these systems [3], [4], [5], [1] is restricted so far to primitive behavior patterns.

We argue that better results could be achieved along the lines of embodied AI [6], [7] or morphological computation [8], [9], [10] —an active field of research with many impressive results, opening new perspectives for both robot control and our understanding of human sensorimotor intelligence [11]. This paper presents an approach that includes the 
world - i. e. body plus environment - more actively and more systematically in the control process than current embodied approaches. By inverting the roles of the controller and the controlled, the world becomes not only "its own best model" (Rodney Brook's idea) but leverages it to "its own best controller" in a metaphorical meaning without aiming at actual optimality. This paper will both develop this idea and outline some of the most intriguing consequences. We attempt to root the idea that "the body shapes the way we think" [6] deeper and more systematically in the physical world, making the latter the actual creator of the robot's way of acting and potentially thinking.

The core of the proposed control algorithm, introduced in Sect. II is a very simple sensorimotor mapping that adapts rapidly according to a modified differential Hebbian-type learning rule [12]. Specific behavior self-organizes in the interaction of the robot, its environment and the dynamics of the controller. This happens without prior knowledge of the system under control. We report on the application of our control framework to a tendon-driven arm-shoulder system in Sect. III In Sect. IV] we show how the robot may discover dynamical affordances of the world by physically interacting with objects which are presented and analyzed here for the first time.

\section{CONTROL STRUCTURE AND SYNAPTIC PLASTICITY}

The control framework is generic and does not use specific knowledge about the system under control. It was previously demonstrated in simulated robots that this control scheme leads to coordinated and purposeful behaviors [12]. In this paper, we consider a tendon driven robotic arm with 9 actuators: 6 in the shoulder, 2 in the elbow and one effecting both. These robots are driven by artificial muscles simulated by tendon-strings that are wind up on a motor spindle and are suspended by springs, see Fig. 2(b). Each muscle $i$ is controlled by a target length $y_{i}$ of the tendon and provides a sensor value $x_{i}$ comprised of the actual tendon length $l_{i}$ combined with the spring compression $f_{i}$,

$$
x_{i}=l_{i}+\beta f_{i}
$$

where $\beta$ regulates the integration of the spring compression/force. We use $\beta=1$ throughout the experiments. The spring force $f$ will be in the interval $[-\alpha, 1-\alpha]$ where $\alpha$ is the initial pretension (here $\alpha=0.1$ ). First there is an initialization procedure where the arm is manually put in a central pose and force control is used to make $f_{i}=0, \forall i$ to create the pretention on the tendons. In this situation the lengths $l_{i}=0$ are set to zero. In general $l$ is normalized to the interval $[-1,1]$, where values correspond to shorter tendon length. Afterwards the plant is set into position control for the tendon length as mentioned above.

\section{A. Controller network and synaptic dynamics}

The theme of our work is structural simplicity, relying upon the paradigm that complex behavior may emerge from the interaction of a simple neural circuitry with the complex external world. Specifically, the controller is a network of rate-coded neurons transforming sensor values $x=$ $\left(x_{1}, x_{2}, \ldots, x_{n}\right)$ into motor commands $y=\left(y_{1}, y_{2}, \ldots, y_{m}\right)$ (target tendon lengths). In the application, a one-layer feedforward network is used, described as

$$
y_{i}=\tanh \left(\sum_{j=1}^{n} C_{i j} x_{j}+h_{i}\right)
$$

for neuron $i$, where $C_{i j}$ is the synaptic connection strength to input $j$ and $h_{i}$ is the bias term, which is set to zero in this work $\left(h_{i}=0\right)$. The motor commands are thus confined to the interval +1 and -1 . The setup is displayed in Fig. 1

Let us assume the robot has a basic understanding of the causal relations between actions and sensor values. In our approach, this is realized by an inverse model which approximately relates the current sensor values $x^{\prime}$ back to its causes, the preceding motor commands $y$. The model will reconstruct (the efference copy) $\tilde{y}$ with a certain mismatch $\delta y$. Formulated in terms of the rates of change, we write

$$
\tilde{\dot{y}}=F\left(\dot{x}^{\prime}\right)
$$

with $F$ representing the inverse model function. We use a linear model as

$$
\tilde{\dot{y}}=M \dot{x}^{\prime}
$$

where $M$ is a unit matrix where $m=n$ in the special case of Myorobotics arm. We also use a delayembedding setting of the sensor values resulting in $x(t)=$ $\left(x_{1}(t), x_{2}(t), \ldots, x_{m}(t), x_{1}(t-d), x_{2}(t-d), \ldots, x_{m}(t-d)\right)$ where $d$ is the time delay and $n=2 m$. In this case we use

$$
M_{i j}=\left\{\begin{aligned}
1 & \text { for } i=j \\
-1 & \text { for } i=m+j \\
0 & \text { otherwise, }
\end{aligned}\right.
$$

which is a unit matrix for the direct sensor to motor mapping and an negative unit matrix for the delay sensor to motor mapping. This setup is most adequate for oscillations with a period of $2 d$. As a helpful modification of the original approach [12], we introduce a normalization factor into the update rule by defining

$$
\hat{\dot{x}}=\frac{1}{\|\dot{x}\|^{2}+r} \dot{x}
$$

(note that $\hat{\dot{x}}$ is not a unit vector) where $\|\dot{x}\|^{2}=\sum_{i} \dot{x}_{i}^{2}$ and $r$ is a regularization term which may range from 0.1 to a minimal value determined by the discretization of the sensor values. However, in practice, a too small $r$ leads to an overly strong influence of very small velocities in case of inactivity.

In these terms, the modified differential extrinsic plasticity (DEP) rule is formulated as

$$
\tau \Delta C_{i j}=\tilde{\dot{y}}_{i} \hat{\dot{x}}_{j}-C_{i j}
$$

where $\tau$ is the time scale for this synaptic dynamics and $-C_{i j}$ is a damping term. Because of the normalization introduced below, we do not need an additional scaling factor for the decay time. In the experiments, $\tau$ is of the order of 1 second. Equation (7) reveals the similarity to 

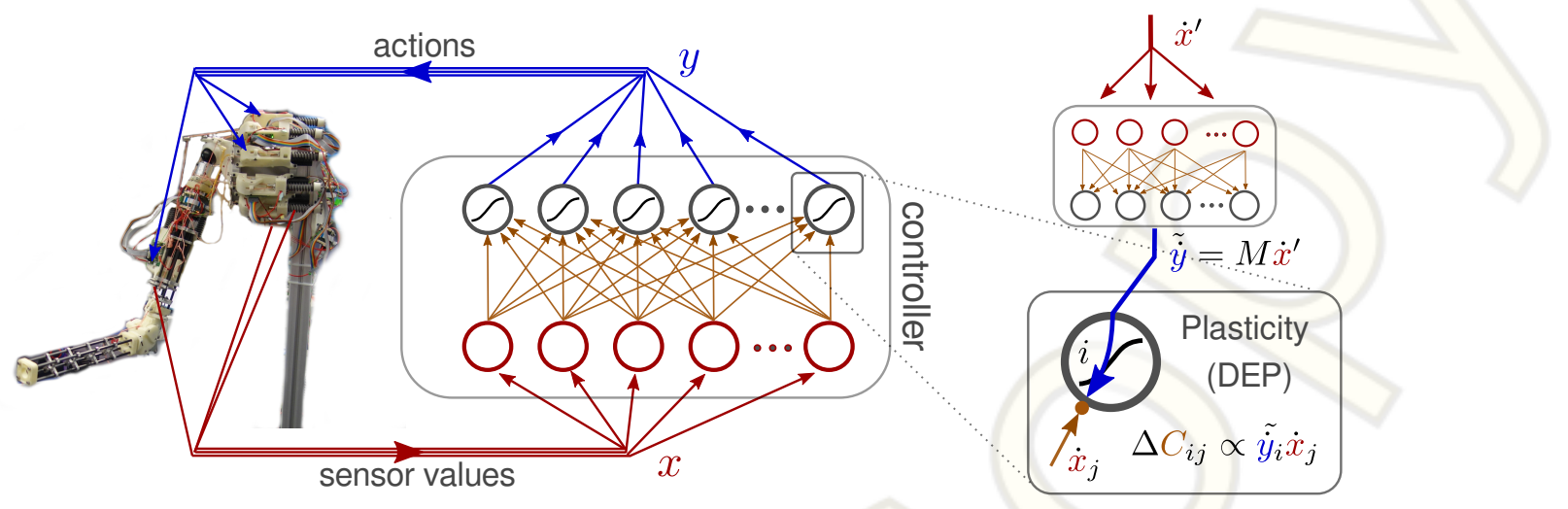

Fig. 1. Neural controller network connected to the Myorobotic arm. The inset on the right illustrates the synaptic plasticity rule, called differential extrinsic plasticity (DEP) [12]. It is driven by a modified differential Hebbian law, multiplying the time derivatives of the incoming sensor values $\dot{x}$ with the virtual motor values $\tilde{\dot{y}}$, which are generated by the inverse model Eq. (4) from the next input's derivative $\dot{x}^{\prime}$. In the case of the arm the inverse model is essentially a one-to-one mapping of sensor to motor values.

differential Hebbian learning, which has the terms $\dot{y}_{i} \dot{x}_{j}$. Recently, differential Hebbian learning was demonstrated to lead to learning of certain reflexes from spontaneous motor activity in muscle driven devices [13].

As in [12], we introduce an appropriate normalization of the synaptic weights $C$ and an empirical gain factor $\kappa \sim 1$. In practice, we use an individual normalization of the synaptic vector of each neuron, i. e. replace Eq. (2) with

$$
y_{i}=\tanh \left(\sum_{j=1}^{n} \kappa \gamma_{i} C_{i j} x_{j}+h_{i}\right)
$$

where $\gamma_{i}=\frac{1}{\sqrt{\sum_{j} C_{i j}^{2}}}$. The empirical factor $\kappa$ regulates the overall feedback strength in the sensorimotor loop. If chosen in the right range external perturbations contained in $\tilde{\dot{y}}$ get amplified to initiate and maintain an active behavior.

Note, that a behavior can become stationary if it reproduces the controller matrix $C$ with the sliding average over $\tilde{\dot{y}} \hat{\dot{x}}^{\top}$, such that $\Delta C$ in Eq. (7) is zero on average. This is the case for harmonic oscillations, see also [14].

This controller network may appear utterly oversimplified. Commonly, and in particular in classical robot control, a certain behavior is seen as the execution of a plan devised by the brain. This would require a highly organized internal brain dynamics, which could never be realized by the simple one-layer network. However, in this paradigm, the essential new feature is the irreducible unity of the controller and the controlled.

When embedding our controller, see Fig. 1, into the sensorimotor loop, a meta-system - consisting of the mechanical system, the controller with its sensor driven synaptic dynamics, and the energy supply - is created displaying a rich behavioral spectrum like limit cycle attractors, long lived transients, and fixed point flows generating pseudorandom sequences of poses and a high responsiveness to the dynamics of the outside world. This has been observed already in [12] and will be corroborated by the experiments reported below. (a)

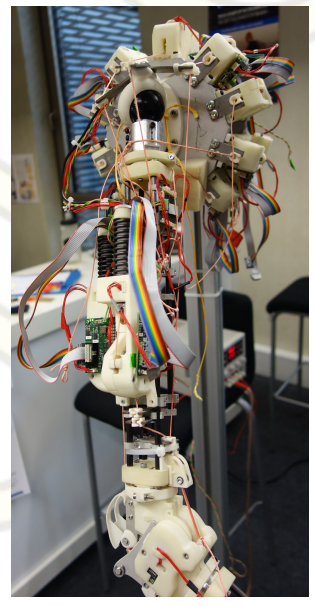

(b)

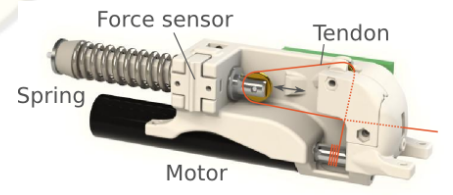

(c)

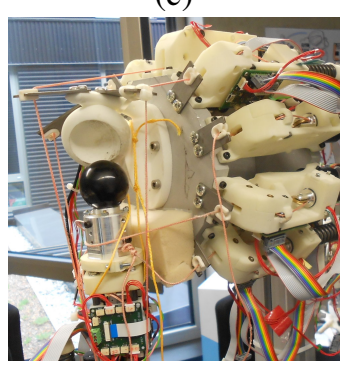

Fig. 2. Myorobotic arm (a) with 9 muscles and a ball shoulder joint, a single muscle element (b), and a dislocated shoulder (c). The dislocation happens immediately as soon as the tendons are getting slack.

\section{UNFOLDING BEHAVIORS}

The above defined controller was used in the experiments with a tendon driven arm-shoulder system from the Myorobotics toolkit, see Fig. 2 .

\section{A. Peculiarities of muscle-tendon driven systems}

There are a number of features which make the muscletendon driven (MTD) systems different from classical robots having revolute joints with direct motor control, i.e. the motor positions directly translate into joint angles and into poses. The most obvious effects stem from the properties of the tendons themselves: they can get slack, wrap or even tangled. These effects make it hard to predict the joint positions from the geometry and the motor positions. To reduce the difficulty and allow for a defined force transmission a permanent tension on the tendons has to be kept, which in turn poses another problem: The tension can only be achieved by tightening each tendon up against all the others, each individual tension being reported by the spring 
compression. This means that (i) there are infinitely many combinations of tension forces for a single arm pose and (ii) that the action of a single motor will be reflected in a change of spring compression of all other muscles. In other words, actuating a single muscle is reflected by a pattern of sensory stimulation-a whole-body answer.

Furthermore, the combination of friction and muscle-pose ambiguity leads to a hysteresis effect. After driving the arm by a sequence of motor commands from the current pose to new pose and then back by reversing the motor commands one ends up in a different pose and muscle configuration than the starting one. In general, this makes the translation of a kinematic trajectory for the arm into motor programs extremely difficult, even more so if there are loads and high velocities involved. Also, the classical approach of learning a model by motor babbling becomes illusory. These problems are partially circumvented when using the whole system dynamics itself for finding the control signals as seen below.

\section{B. Self-regulation}

Let us now have a look at the main characteristics of the control approach, which is also discussed in [14]. This paper goes into more detail and present new experiments, see Tab. If for an overview and videos. At first, we observed the self-regulation into a working regime where the tendons are kept under tension even in very rapid motions with notable loads. This is very important as it guarantees the signals from the controller to be executed in a definite way. As a result, in all experiments we never had to face a shoulder dislocation, see Fig. 2(c), which may happen promptly if tendons are becoming slack. This is even more astonishing as this sensible working regime emerges from the controller without any additional tuning or calibrating the system. For that, the integration of the forces into the sensor values Eq. (1) is important. We did not study it systematically yet and expect other configurations to work as well. In the experiments we used the following parameter settings: $\kappa=0.5, \tau=1 s, d=0.5 s$, a time distance between $x$ and $x^{\prime}$ of $0.08 s, r=10^{-3}$ and a update frequency of the control loop of $100 \mathrm{~Hz}$.

\section{Manipulability}

The dominance of the world in generating the controller signal, see Eq. (4), makes the controlled system manipulable by external forces. The point is that any externally applied forces change the sensor values via the changing spring compression, see Eq. (1) and thus changes both factors of the update equation Eq. (7). This effect integrates manipulative influences-like a physical human-robot interactioninto the sensor values and thereby, via $C$, in the behavior generation.

For instance, the arm can always be stopped by applying a force by hand. The reason is not at all that the motors are too weak. Instead, $\dot{x}=0$ is a fixed point of the dynamics of the meta-system to which it relaxes if the mechanical degrees of freedom are frozen manually. Moreover, the system can be entrained by manual interaction into specific behaviors. We

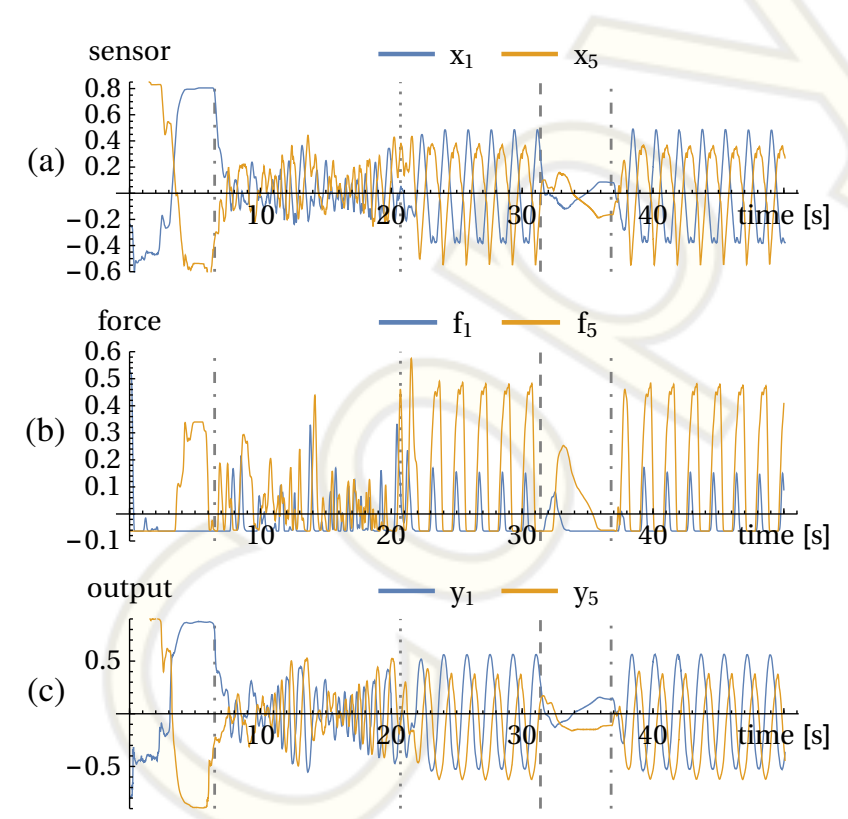

Fig. 3. Handshake experiment. (a) sensor values $x$, (b) forces $f$, and (c) motor values $y$ for channels 1 and 5. Events: $6 \mathrm{~s}$ : operator is grasping the arm and starts the handshake; $21 \mathrm{~s}$ : freezing of parameters and release; $31 \mathrm{~s}$ : bringing arm into resting position, it stays there from 35.5 to $37 \mathrm{~s}$ where it got perturbed. See also corresponding Video 2

demonstrate this in the handshake experiment, see Video 2. where the user is trying to move the arm in a periodic pattern. Besides the possibility to train a robot in this way, the most interesting point is the subjective feeling that comes about when interacting with the robot. In the beginning of such an interplay, the robot seems to have a will of its own as it resists the motions the user is trying to impose. But after a short time the robot more and more is following the human. Eventually, by freezing the controller weights the robot can uphold the imposed motion, see Fig. 3 . If the parameters are not frozen the "negotiated" joint motion pattern is possibly left if the human quits the loop. In fact, in the experiments, we observed by letting different people interact with the robot that a "compliant" user is intrigued to follow the system as much as its own intentions, ending up in an orchestrated human-machine dynamical pattern.

\section{Emerging modes}

The meta-system is particularly akin to periodic motions, i. e. there is a plenitude of latent limit cycle attractors which, metaphorically speaking, wait for their excitation. This can be seen for instance when suspending a weight (the bottle) from the tip of the arm. Video 3 demonstrates how latent velocity correlations are being amplified to end up in stable circular motion patterns of the weight. The experiment starts in a situation where the motor activities have settled to rest, interrupted by occasional bursts so that the bottle is excited to some minor pendulum motion. These oscillations directly exert physical forces on the arm which propagate via the springs into the sensor values, see Eq. (1), and eventually into the $C$ matrix which governs the behavior. To illustrate this effect, Fig. 4 displays the forces measured by the sensors 
TABLE I

VIDEOS FOR THE INDIVIDUAL EXPERIMENTS AVAILABLE AT playfulmachines.com/MyoArm-2

\begin{tabular}{|c|c|c|c|}
\hline Title & Description & Sect. & Vid./Link \\
\hline Overview & Compiled clip of all experiments & (2) & Video 1 \\
\hline Handshake & Human robot interaction by manually imposing a periodic movement & III-C & Video $\overline{2}$ \\
\hline Bottle swing & Excitation of a circular pendulum mode & III-D & Video 3 \\
\hline Bottle swing measure & Motors are stopped. Recording spring forces of swinging susper & III-D & V1deo 4 \\
\hline Shaking vertically & half filled bottle at tip of the arm: shaking mainly along bottle's & III-D & Video \\
\hline Shaking horizontally & Same as above but with horizontal attachment & III-D & V1deo \\
\hline Rotating wheel & Arm attached to a revolvable bar/wheel & IV-.0.a & Video 7 \\
\hline Rota & Para & IV-.0.a & Video 8 \\
\hline Rotating & Diffe & IV-.0.a & Video 9 \\
\hline Wiping table & 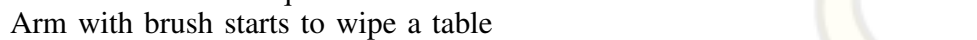 & IV-.0.b & Video 10 \\
\hline Wiping table modes & Different wiping patterns from reloaded & IV-.0.b & V1deo \\
\hline Free & o external forces applied: pseudo-random sequences of & & Video \\
\hline
\end{tabular}
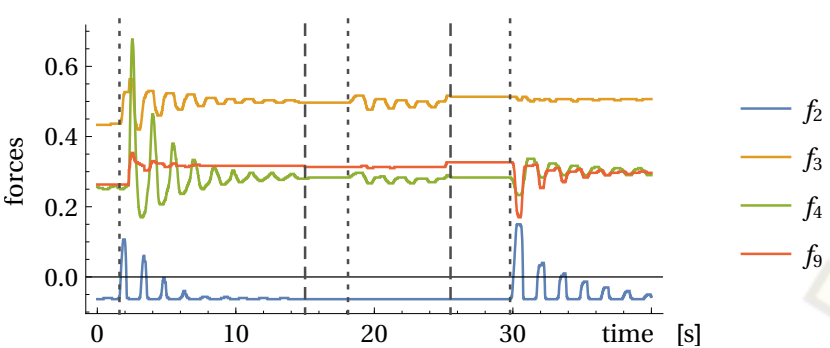

Fig. 4. Force measurements with swinging bottle but without active arm movements for muscles 2, 3, 4 and 9 (for clarity). Dotted lines indicate when the bottle was manually set into motion and at dashed lines it was stopped, see Video 4

when the bottle swings but the motors are stopped. Also relatively small movements of the swinging bottle can be detected. In the dynamics, now with enabled motors, this may lead to self-amplification of latent pendulum modes as observed in the experiments. These findings elucidate how the pendulum as a physical subsystem is guiding-by its internal dynamics - the meta-system into a resonant state, i. e. a whole-system mode with defined frequency. When analyzing the phase relation between measured force and driving signal (motor commands), see Fig. 5, it becomes evident that initially the bottle and arm are not in a fixed phase relation and only become coherent once the swinging mode is excited from $30 \mathrm{~s}$ on.

An indicator for the oscillations is found in the complex eigenvalues of the linearized system dynamics as displayed in Fig. 6. During the swinging mode, we find only 1 pair of significantly non-zero complex eigenvalues representing the main oscillatory component.

In a series of experiments we attached a bottle half-filled with water to the tip of the arm in either horizontal or vertical orientation. Both Video 5 and Video 6 show the emergence of stable shaking modes. Again, we see how the metasystem may become resonant with the internal dynamics of a subsystem, if the latter provides correlations over space and time. This is the case for instance when the water is hitting either the walls or top and bottom of the bottle. These impacts cause a reaction of the springs and hence of the sensor values, which may increase correlations in the

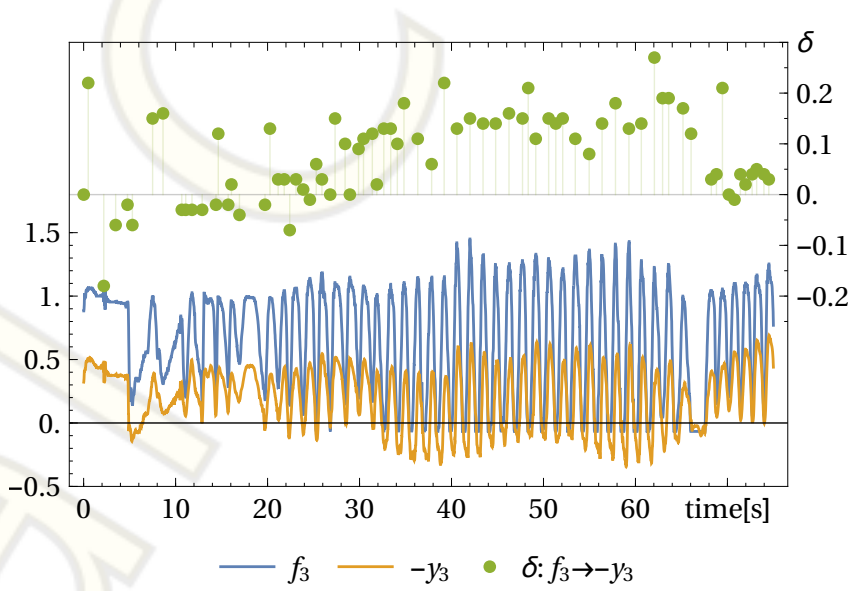

Fig. 5. Bottle swing: Phase-relation between force sensors and contro signal of muscle 3 . The measured force $f$ and the control signal $y$ follow a similar trajectory with inverted sign (note $-y$ ). The phase difference $\delta$ (right axis in [s]) between force and motor value. At second 66 the string of the bottle was shortened, see Video 3

synaptic dynamics resulting in enhanced motions of the arm in coherence with these signals.

\section{Discovering OBJECT AFFORDANCES AND TOOL USE}

The most interesting results are obtained if the robot is interacting with objects having a certain functionality of their own. We claim that by this interaction and the emerging self-amplification effects, the robot may discover dynamical affordances of the world.

a) Rotating a wheel: One striking example is the robot arm connected to a wheel. In terms of Gibson's [15] theory of affordances a wheel affords rotating, in the same sense as a chair affords sitting or a knob affords turning. With our controller, the robot "discovers" such affordances without any knowledge of the physics of the system and/or specific motivation for doing just that task. It only has a bias towards rotational movements, as these are possible fixed points of the parameter dynamics. Earlier [12] it was demonstrated that DEP leads to spontaneous coordinated rotation of wheels by a humanoid robot in simulation. Here, the end of the robotic arm is attached to a crank of a wheel, implemented as a revolvable bar with weights for giving it some moment 


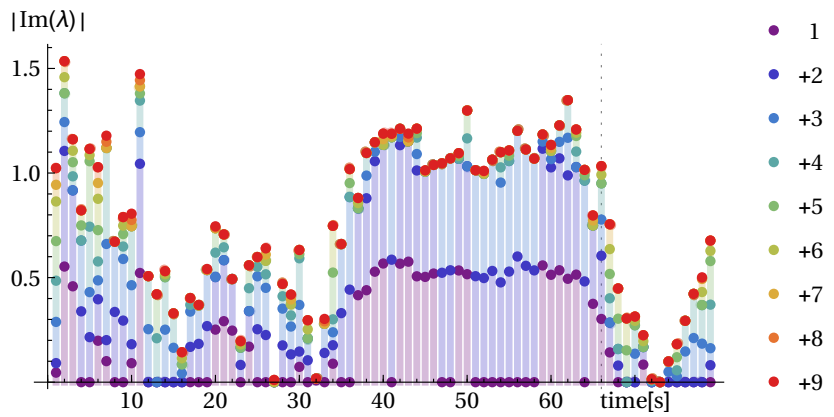

Fig. 6. Self-excitation of an oscillatory mode. Displayed are the absolute imaginary parts of the eigenvalues of the linearized system dynamics (Jacobian $L=C M^{-1}$ ) (averaged over $1 \mathrm{~s}$ ) and cumulatively plotted $(1,1+2,1+2+3, \ldots)$. During the pronounced oscillation between 35 and $68 \mathrm{sec}$ there is one pair of dominant complex eigenvalues.

of inertia. In Video 7, initially the connection between the arm and the wheel was rather loose so that for small movements there is not reaction from the rotation of the wheel. After improving this connection, an initial push by the experimenter was sufficient to excite a rotation mode, that persists over time and is stable under mild perturbations. It is as if the controller "understood" how to rotate the wheel, although it is just the result of force exchange and dynamics of the meta-system. When positioning the wheel in parallel to the arm, the modes were emerging even more readily as seen in Video 8 . Moreover, the system can immediately be switched between forward and backward rotation mode. This is possible because the time-scale of the synaptic plasticity is so fast (in the order of one second) that the new dynamics is quickly propagating into the controller via the plasticity rule. However, during these rotational modes the internal dynamics (Eq. (7)) is approximately at a fixed point. By changing the time-delay $d$ of the delay-sensors (see above Eq. (5)) the frequency of rotation can be adjusted, see Fig. 7 and Video 9. Also the amplitude gets decreased, which is most likely due to limited motor speed. Note that the timedelay prescribes a preferred frequency that, however, is not necessarily followed. The controller can generate oscillations by different means, either by coupling to the delay sensors or by implementing a rotation matrix, as in Fig. 6 , in which case any frequency can be implemented. We have observed both in the experiments.

b) Wiping: In another experimental situation, the robot is equipped with a brush and forced by manual guidance to wipe a table. The table height is about $5 \mathrm{~cm}$ above the initialized resting position. Video 10 demonstrates how, by the combination of the restricting table surface and the manual force, the robot is driven into the two-dimensional wiping mode. Later in this video the robot is forced into a different behavior, which is analyzed in Fig. 8. To illustrate the different wiping modes we plot the phase difference between some sensor channels. The phase values are obtained using the Hilbert transformation of the time series for each channel. Postprocessing is applied to avoid unnecessary $2 \pi$ phase jumps and to smoothen the signal for better visibility. Actually already before manual interaction the meta-system

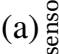

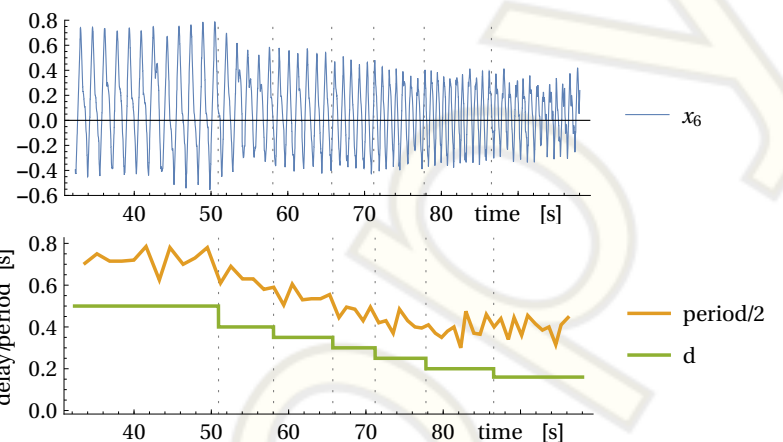

Fig. 7. Rotating the wheel. The frequency of resulting rotation behavior can be changed by changing the delay $d$ of the delay-sensors (Sect. II-A) (a) Sensor trajectory $x_{6}$ (representative for other sensors). (b) Sensor delay $d$ and half oscillation period (in seconds), see Video 9 Dashed lines indicate changes of $d$.

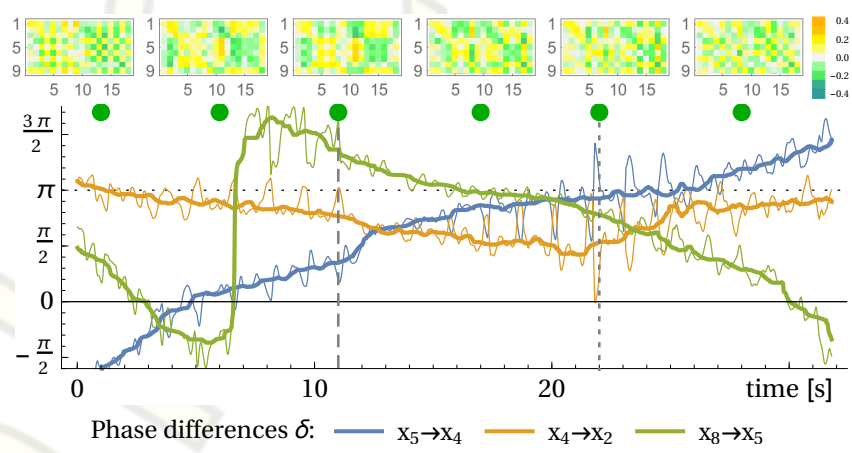

Fig. 8. Learning to wipe a table with a brush. Shown are the phase differences between a selection of sensor values (bottom) and the controller matrices (top) at different points in time indicated by green dots. The thick lines show the sliding median of 2 seconds windows for better visibility. See corresponding Video 10 ( 0 seconds in the plot is at the first cut in the video). From second 11 (dashed line) to 22 (dotted line) the arm was trained to perform a different movement, which persists for a few seconds until the system drifts away.

is in a transient behavior changing the phase relation during the course of time. During interaction (second 11 onward) the changes are initially stronger and get weaker later. After release of the arm by the operator (second 22) the behavior is maintained for a few seconds and then drifts away. The corresponding controller matrices also show a significantly different structure in the course of the experiment.

As seen above, even without interaction the transients into a meta-stable behavior are very long such that different wiping patterns occur. By simply storing the weights $(C)$ of the controller these patterns can be collected into a repertoire. Video 11 shows the recall of and switching between such wiping modes, see Fig. 9 For the transition into a different mode the controller was changed abruptly, nevertheless a smooth transition into the new behavior occurs, suggesting that most static controllers have a large basin of attraction.

\section{SUMMARY AND OUTLOOK}

This paper discusses a novel approach to control of embodied systems. We demonstrated that a simple neural network with the DEP rule, when coupled to a muscle-tendon driven system, can excite a large number of behavioral competences in a self-organized way. In the experiments we 


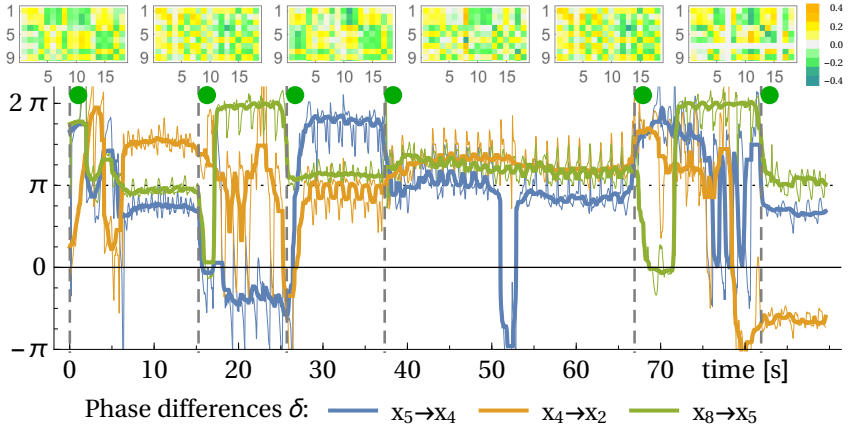

Fig. 9. Recall of different wiping patterns. At vertical dashed lines, a static controller was loaded. Phase differences between a selection of sensor values (bottom) and the controller matrices (top) (times, see green dots). See corresponding Video 11] Observe the transients between the behaviors, which are sometimes long, e. g. $15 \mathrm{sec}$ for controller 4.

actually witnessed the emergence of a new kind of robotics. The novelty can be seen best from the point of view of physics. With the controller in the loop, we have a metasystem consisting of the mechatronic system with controller and energy supply. The nature of that meta-system is ruled by the value of the gain parameter $\kappa$. With $\kappa$ above a critical value, the meta-system was shown to be host to a large variety of behavior patterns which can be excited. These patterns can emerge spontaneously or can be elicited by manual interaction. These patterns can be understood as if the controller tries to identify and amplify the tiny responses from the world outside itself.

In particular, if the robot's body is extended by attaching objects with an internal dynamics of their own, the controller gets in a functional resonance with them. The emerging shaking and pendulum modes were earlier reported examples. This paper analyses them and is devoted to the discovery of object affordances by the robot arm, with the robot at the wheel as the generic example. Behavioral primitives acquired in a self-organized way and exploiting object affordances, i.e. the emergence of tool use, may form pre-requisites for higher-level control, prediction and planning. The bootstrapping of new behavioral modes is an important step for creating truly autonomous systems and to create creativity in cognitive systems. We hope to support these ideas with our future investigations.

All these patterns emerge with great ease and in a natural and elegant way. From the point of view of practical applications, the novelty of this approach can be seen from the fact that existing controller paradigms would have great difficulties to generate any of the observed behavior patterns. Remember that the controller receives nothing else but the sensor signals - the sum of tendon plus spring length, and that it has no knowledge of the physics of the mechanical system. It will be a challenging task for the future to connect this approach with common control paradigms in order to build complex, embodied functional architectures. Inversely, interpreting this controller as a simple approach to generate complex, force-sensitive interactions with the environment, it could also augment the repertoire of classical controllers. Additionally, it may shed light on how biological musculoskeletal systems generate the complex trajectories they use to interact with the environment with an unrivalled flexibility.

As a perspective, the observed compliance of the system to the world's internal dynamics - as demonstrated for instance by the wheel-shows the way to an important generalization: equipping the robot with more sensors that might report the spatial relation of the robot's mechanical degrees of freedom to the structure of the environment, we expect a similar integration of those relations into the emerging behavioral modes. By integrating a camera, this mechanism can even lead to an active exploration of visuomotor coordination, but this is the topic of further studies.

Finally, we expect our approach to be effective not only in softly actuated machines, as it was done here, but also in structurally soft robots because the exploitation of body dynamics is even more important there.

\section{ACKNOWLEDGMENT}

RD thanks for the hospitality at the Max-Planck-Institute and for helpful discussions with Nihat Ay and Keyan Zahedi.

\section{REFERENCES}

[1] Y. Nakanishi, S. Ohta, T. Shirai, Y. Asano, T. Kozuki, Y. Kakehashi, H. Mizoguchi, T. Kurotobi, Y. Motegi, K. Sasabuchi, et al., "Design approach of biologically-inspired musculoskeletal humanoids," International Journal of Advanced Robotic Systems, vol. 10, 2013.

[2] S. Wittmeier et al., "Toward anthropomimetic robotics: development, simulation, and control of a musculoskeletal torso," Artificial life vol. 19, no. 1, pp. 171-193, 2012.

[3] V. Potkonjak, B. Svetozarevic, K. Jovanovic, and O. Holland, "Anthropomimetic robot with passive compliance-contact dynamics and control," in Proc. Control \& Automation (MED). IEEE, 2011, pp. $1059-1064$

[4] V. Potkonjak, K. M. Jovanovic, P. Milosavljevic, N. Bascarevic, and O. Holland, "The puller-follower control concept in the multi-jointed robot body with antagonistically coupled compliant drives," in IASTED international conference on robotics, 2011, pp. 375-381.

[5] M. Jäntsch, S. Wittmeier, K. Dalamagkidis, and A. Knoll, "Computed muscle control for an anthropomimetic elbow joint," in Intelligent Robots and Systems (IROS), 2012 IEEE/RSJ International Conference on. IEEE, 2012, pp. 2192-2197.

[6] R. Pfeifer and J. C. Bongard, How the Body Shapes the Way We Think: A New View of Intelligence. Cambridge, MA: MIT Press, 2006.

[7] R. Pfeifer and C. Scheier, Understanding intelligence. Boston: MIT Press, 1999.

[8] H. Hauser, A. J. Ijspeert, R. M. Füchslin, R. Pfeifer, and W. Maass, "The role of feedback in morphological computation with compliant bodies," Biological Cybernetics, vol. 106, no. 10, pp. 595-613, 2012.

[9] R. Pfeifer and G. Gómez, "Morphological computation - connecting brain, body, and environment," in Creating Brain-Like Intelligence. Springer, 2009, pp. 66-83.

[10] C. Paul, "Morphology and computation," in Proc. Int. Conf. on Simulation of Adaptive Behavior. MIT Press, 2004, pp. 33-38.

[11] R. Pfeifer, M. Lungarella, and F. Iida, "The challenges ahead for bioinspired 'soft' robotics," Commun. ACM, vol. 55, no. 11, pp. 76-87, 2012.

[12] R. Der and G. Martius, "Novel plasticity rule can explain the development of sensorimotor intelligence," Proceedings of the National Academy of Sciences, vol. 112, no. 45, pp. E6224-E6232, 2015.

[13] H. G. Marques, F. Imtiaz, F. Iida, and R. Pfeifer, "Self-organization of reflexive behavior from spontaneous motor activity," Biological cybernetics, vol. 107, no. 1, pp. 25-37, 2013.

[14] R. Der and G. Martius, "Self-organized control for musculoskeletal robots," 2016, arXiv preprint http://arxiv.org/abs/1602.02990

[15] J. J. Gibson, The theory of affordances, in Perceiving, Acting, and Knowing. Towards an Ecological Psychology. Hoboken, NJ, 1977. 